\title{
Protocol
}

\section{Protocol for a prospective multicenter intervention study (URivo study) assessing biomarkers in patients with previously treated advanced clear cell renal cell carcinoma receiving nivolumab}

\author{
Hideaki Miyake $^{1,2 *}$, Ken-ichi Harada ${ }^{1}$, Yuto Matsushita ${ }^{2}$, Nobuyuki Hinata ${ }^{1}$, \\ Haruhiko Sugimura ${ }^{3}$, Masato Fujisawa ${ }^{1}$
}

\author{
${ }^{1}$ Division of Urology, Kobe University Graduate School of Medicine, Kobe, Japan \\ ${ }^{2}$ Department of Urology, ${ }^{3}$ Department of Tumor Pathology, Hamamatsu University School of Medicine, Hamamatsu, \\ Japan
}

Received: 08 January 2019

Accepted: 07 February 2019

\author{
*Correspondence: \\ Dr. Hideaki Miyake, \\ E-mail: hmiyake@hama-med.ac.jp
}

Copyright: (c) the author(s), publisher and licensee Medip Academy. This is an open-access article distributed under the terms of the Creative Commons Attribution Non-Commercial License, which permits unrestricted non-commercial use, distribution, and reproduction in any medium, provided the original work is properly cited.

\begin{abstract}
Background: In recent years, immune checkpoint inhibitors have been introduced into routine clinical practice for treating patients with a wide variety of malignant tumors, including advanced renal cell carcinoma (aRCC), resulting in the significant improvement of the prognosis of these patients. However, a reliable biomarker prediciting the clinical course in patients receiving nivolumab has not yet been developed; accordingly, the URivo study was planned to investigate the significance of various candidate biomarkers for aRCC patients treated with nivolumab.

Methods: This was designed as a prospective multicenter intervention study, and will include a total of 200 aRCC patients who are scheduled to receive nivolumab followed by treatment with either 1 or 2 tyrosine kinase inhibitors (TKIs). Using resected tumor tissues and serum samples prior to the introduction of nivolumab, the following assessments will be conducted: programmed death ligand-1 (PD-L1) and PD-L2 gene copy number gains by fluorescence in situ hybridization, serum concentrations of PD-L1 and PD-L2 by enzyme-linked immunosorbent assay, and expression of several proteins involved in apoptosis, epithelial-mesenchymal transition, signal transduction and immune reaction by immunohistochemical staining. The outcomes of these assays will be evaluated focusing on the association with the response to nivolumab, overall survival, progression-free survival and disease-specific survival.

Conclusions: The significance of various types of candidate biomarker, particularly PD-L1 and PD-L2, will be intensively investigated in this study, and this may offer unique information to determine the optimal indication of nivolumab for aRCC patients following the failure of TKIs.

Trial Registration: UMIN000030400; registered April 1, 2018.
\end{abstract}

Keywords: Advanced clear cell renal cell carcinoma, Nivolumab, Biomarker, PD-L1, PD-L2

\section{INTRODUCTION}

During the past 10 years, a paradigm shift has occurred in the field of systemic therapy for advanced renal cell carcinoma (aRCC) due to the introduction of various novel agents developed based on the molecular mechanisms mediating the progression of RCC. ${ }^{1}$ These agents, which target vascular endothelial growth factor (VEGF) or mammalian target of rapamycin (mTOR), have significantly contributed to favorable clinical outcomes in patients with aRCC, particularly those sequentially receiving multiple agents. ${ }^{2,3}$ However, some 
aRCCs are inherently resistant to molecular-targeted agents, and almost all aRCCs will ultimately acquire phenotypes resistant to these agents over time, resulting in limitation of the overall clinical benefit; thus, it is recognized that the development of new therapies with different mechanisms of action is necessary to further improve the prognosis of aRCC patients. ${ }^{3,4}$

In recent years, the therapeutic strategy for aRCC markedly changed with the advent of new options that target immune escape by tumor cells; that is, antibody blockade of major molecules involved in immune checkpoint pathways, including programmed death-1 (PD-1), PD-ligand 1 (PD-L1) and cytotoxic Tlymphocyte antigen 4 (CTLA-4), has been shown to promote marked antitumor activities against a wide variety of malignant tumors, including RCC. ${ }^{5}$ Of these immune checkpoint inhibitors, nivolumab, a human IgG4 PD-1 antibody that blocks the interaction between PD-1 and its ligands, PD-L1 and PD-L2, was initially approved by the Food and Drug Administration based on data from the CheckMate 025 clinical trial. ${ }^{6}$ In this trial, comparing the efficacies between nivolumab and everolimus in aRCC patients who had previously received antiangiogenic therapy, significantly longer overall survival (OS) and fewer severe adverse events (AEs) were shown in the nivolumab group compared with everolimus group. ${ }^{7}$ Accordingly, nivolumab is currently regarded as the standard agent for aRCC patients who failed prior antiangiogenic agents in major clinical guidelines. ${ }^{8}$

Although immune checkpoint inhibitors have been widely accepted as promising systemic agents for the treatment of advanced malignant tumors, response rates are variable, and immune-related AEs associated with the use of these agents can occur. ${ }^{9}$ Therefore, intensive efforts have been focused on identifying reliable biomarkers that may predict the clinical course of patients treated with immune checkpoint inhibitors. ${ }^{10}$ As for antiPD-1 antibodies, PD-L1 expression in tumor tissues may be a useful biomarker based on its action mechanism; however, the predictive role of PD-L1 expression in the outcomes of treatment with anti-PD-1 antibodies remains unclear, particularly in aRCC patients. ${ }^{11}$ In fact, the benefit of nivolumab was suggested irrespective of PDL1 expression in the CheckMate 025 clinical trial. $^{7}$

Considering the lack of a useful biomarker for the selection of aRCC patients receiving nivolumab, the URivo study was designed to investigate the value of several candidate biomarkers for aRCC patients treated with nivolumab. In particular, it may be the first study to comprehensively evaluate the predictive value of PD-L1 and PD-L2 expression by multiple assays, including immunohistochemical staining, fluorescence in situ hybridization (FISH) and enzyme-linked immunosorbent assay (ELISA), in these patients.

\section{METHODS}

\section{Study design}

The URivo study is an ongoing multicenter prospective open-label intervention single-arm study to identify reliable biomarkers in previously treated aRCC patients who will be treated with nivolumab. This study will evaluate whether the findings of several candidate biomarkers, which are described in detail below, are significantly correlated with the clinical outcomes of aRCC patients.

\section{Endpoints}

The primary endpoint of the URivo study is to investigate the impact of the findings of candidate biomarkers on the best overall response to nivolumab in the included aRCC patients. The tumor response to nivolumab will be assessed according to the response evaluation Criteria in Solid Tumors (RECIST) version 1.1.

The secondary endpoint is to assess the association between the findings of candidate biomarkers and prognostic outcomes, including OS, progression-free survival (PFS) and disease-specific survival (DSS), in these patients. Definitions of prognostic outcomes are as follows: OS, the time from the initiation of nivolumab to death due to any cause; PFS, the time from the initiation of nivolumab to first-demonstrated RECIST-defined tumor progression or death due to any cause; and DSS, the time from the initiation of nivolumab to death due to the progression of RCC.

\section{Study population}

Patients with aRCC, who were previously treated with either 1 or 2 TKIs and are going to receive nivolumab, are eligible for enrollment in the URivo study. After a full explanation of the study protocols, written informed consent from each participant will be obtained. Detailed eligibility and exclusion criteria for this study are listed in below.

\section{Inclusion criteria}

Inclusion criteria were unresectable or metastatic renal cell carcinoma; pathologically confirmed dominant component of clear cell carcinoma; previous history of treatment with 1 or 2 tyrosine kinase inhibitors; tissue specimens can be provided; measurable diseases according to RECIST version 1.1; major organ functions fulfilling the following criteria: neutrophil count $\geq 1,500 / \mathrm{mm}^{3}$, platelet count $\geq 75,000 / \mathrm{mm}^{3}$, hemoglobin $\geq 9.0 \mathrm{~g} / \mathrm{dl}$, aspartate aminotransferase $\leq 78 \mathrm{IU} / \mathrm{l}$, alanine aminotransferase $\leq 85 \mathrm{IU} / \mathrm{l}$, total bilirubin $\leq 1.5 \mathrm{mg} / \mathrm{dl}$, creatinine $\leq 2.0 \mathrm{mg} / \mathrm{dl}$; Age $\geq 20$ years upon enrolment; Eastern Cooperative Oncology Group performance status 0 or 1 ; submission of written informed consent to participate in this study 


\section{Exclusion criteria}

Exclusion criteria were previous history of treatment with immune checkpoint inhibitors, including nivolumab; active infection; malignant tumors other than renal cell carcinoma; severe acute or chronic diseases other than infection or malignant tumors; woman who is pregnant or nursing; mental disease preventing participation in this study; intolerance to nivolumab; inappropriate characteristics for participating in this study other than those listed above

\section{Study procedures}

The flowchart of the URivo study is presented in Figure 1. After obtaining informed consent by the attending physician, patients will undergo screening examinations, and their eligibility to be included in this study will be evaluated. Screening examinations will consist of patients' characteristics (gender, age, height, body weight, complications, Eastern Cooperative Oncology Group performance status, Union for International Cancer Control TNM classification, Memorial SloanKettering Cancer Center risk classification for previously treated aRCC patients and histopathological information of RCC), blood tests (red blood cell count, hemoglobin, hematocrit, white blood cell count, neutrophil count, platelet count, total protein, albumin, total bilirubin, aspartate aminotransferase, alanine aminotransferase, alkaline phosphatase, lactate dehydrogenase, amylase, blood urea nitrogen, creatinine, uric acid, creatine phosphokinase, C-reactive protein, blood glucose, sodium, potassium, chlorine and calcium) and radiological examinations for the assessment of target lesions (enhanced computed tomography or magnetic resonance imaging). ${ }^{12}$

Then, screened information on each patient judged to be eligible will be entered in the electronic data capture (EDC) system of this study, and case registration will be regarded as completion by re-conformation through the EDC system. Within 28 days after the registration, it will be necessary to initiate the treatment with nivolumab at a dose of $3 \mathrm{mg} / \mathrm{kg}$ as an intravenous infusion every 2 weeks, until disease progression or the development of intolerable AEs. ${ }^{7}$ Dose interruption of nivolumab based on the proper use guide (https://www.opdivo.jp/ drug_info_files/drug_info/opdivo/tekisei/20000157/guide _rcc.pdf) will be permitted.

In addition, each patient will provide a $20 \mathrm{~mL}$ blood sample and formaldehyde-fixed, paraffin-embedded tumor tissue sample before starting treatment with nivolumab, and these samples will be stored for the future investigation of biomarkers.

\section{Follow-up schedule}

Following the initiation of treatment with nivolumab, each participant is required to visit the attending physician every 2 weeks for the administration of nivolumab and the assessment of AEs by blood examinations. Radiological examinations for the assessment of target lesions will be performed every 8 weeks within 24 weeks after starting nivolumab therapy, and be continued every 12 weeks thereafter.

\section{Biomarker analyses}

The URivo study includes the following biomarker assays using tissue and serum specimens obtained from participants, that will be performed while blinded to their clinicpathological data.

\section{FISH}

FISH analyses will be performed using tissue specimens to investigate copy numbers of PD-L1 and PD-L2 genes, as previously described. ${ }^{13}$

\section{ELISA}

Concentrations of PD-L1 and PD-L2 in serum samples will be measured using ELISA kits (ThermoFisher Scientific, Waltham, MA, USA) according to the manufacturer's instructions.

\section{Immunohistochemical staining}

Immunohistochemical staining of tissue specimens will be conducted using antibodies targeting the following proteins, as previously described: ${ }^{14} \mathrm{Akt}$, phosphorylated (p)-Akt (Cell Signaling Technology, Danvers, MA, USA), Bax (Abcam, Cambridge, United Kingdom), Bcl-2 (Dako, Carpinteria, CA, USA), Bcl-xL (Santa Cruz Biotechnology, Santa Cruz, CA, USA), E-cadherin, Ncadherin (Dako), $\beta$-catenin (BD Transduction Laboratories, Franklin Lakes, NJ, USA), CD4, CD8 (Dako), clusterin (Santa Cruz Biotechnology), heat shock protein27 (HSP27), HSP90 (Novocastra Laboratories, Newcastle, United Kingdom), c-Jun N-terminal kinase (JNK), p-JNK (Cell Signaling Technology), Ki-67 (Dako), mitogen-activated protein kinase (MAPK), pMAPK (Cell Signaling Technology), matrix metalloproteinase (MMP)-2, MMP-9 (Daiichi Fine Chemical, Toyama, Japan), Mcl-1 (Santa Cruz Biotechnology), p53 (Novocastra Laboratories), PD-L1, PD-L2 (Dako), PTEN (Proteintech, Rosemont, IL, USA), signal transducers and activation of transcription 3 (STAT3), p-STAT3 (Cell Signaling Technology), Slug, Snail (Abcam), vimentin (Santa Cruz Biotechnology) and ZO-1 (Cell Signaling Technology).

\section{Sample size, follow-up period and power calculations}

The URivo study plans to recruit 200 participants, and the duration of participant recruitment will be 2 years. The follow-up period will be 3 years from enrollment of the final participant. Therefore, the total duration of this study will be up to 5 years. 
In this study, the ratio of responders to non-responders to nivolumab is considered to be 2 to 1 based on the outcomes of the CheckMate 025 study in order to determine an optimal sample size. ${ }^{7}$ When it is estimated that the area under the curve (AUC) of the receiver operating characteristic curve (ROC) for a certain parameter as a predictor of endpoints of this study is 0.50 and 0.65 under the null and alternative hypotheses, respectively, 196 patients have to be included to achieve a power of 0.90 using a significance level of 0.025 based on a one-sided test. Accordingly, a total of 200 participants are scheduled to be included in this study after considering the presence of those who will drop out.

\section{Research organization}

A complete list of principal investigators and their institutions involved in the URivo trial is provided in Table 1.

Table 1: Research organizations.

\begin{tabular}{|c|c|}
\hline Participating institutions & $\begin{array}{l}\text { Principle } \\
\text { investigators }\end{array}$ \\
\hline Hokkaido University & Nobuo Shinohara \\
\hline Hirosaki University & Chikara Ohyama \\
\hline $\begin{array}{l}\text { Iwate Medical University } \\
\text { School of Medicine }\end{array}$ & Wataru Obara \\
\hline Chiba University & Akira Komiya \\
\hline Juntendo University & Shigeo Horie \\
\hline Keio University & Mototsugu Oya \\
\hline $\begin{array}{l}\text { Tokyo Women's Medical } \\
\text { University }\end{array}$ & Kazunari Tanabe \\
\hline Yokohama City University & $\begin{array}{l}\text { Noboru } \\
\text { Nakaigawa }\end{array}$ \\
\hline $\begin{array}{l}\text { Hamamatsu University School } \\
\text { of Medicine }\end{array}$ & Hideaki Miyake \\
\hline Nagoya City University & Noriyasu Kawai \\
\hline Fujita Health University & Ryoichi Shiroki \\
\hline $\begin{array}{l}\text { Shiga University of Medical } \\
\text { Science }\end{array}$ & Akihiro Kawauchi \\
\hline $\begin{array}{l}\text { Kyoto Prefecture University of } \\
\text { Medicine }\end{array}$ & Osamu Ukimura \\
\hline Osaka Medical College & Haruhito Azuma \\
\hline Osaka City University & Satoshi Tamada \\
\hline Kinki University & Hirotsugu Uemura \\
\hline Nara Medical University & $\begin{array}{l}\text { Kiyohide } \\
\text { Fujimoto }\end{array}$ \\
\hline Wakayama Medical University & Isao Hara \\
\hline Kobe University & Masato Fujisawa \\
\hline Tokushima University & $\begin{array}{l}\text { Hiro-omi } \\
\text { Kanayama }\end{array}$ \\
\hline Okayama University & Yasutomo Nasu \\
\hline Hiroshima University & Akio Matsubara \\
\hline Tottori University & Atsushi Takenaka \\
\hline Kyusyu University & Masatoshi Eto \\
\hline Oita University & Hiromitsu Mimata \\
\hline
\end{tabular}

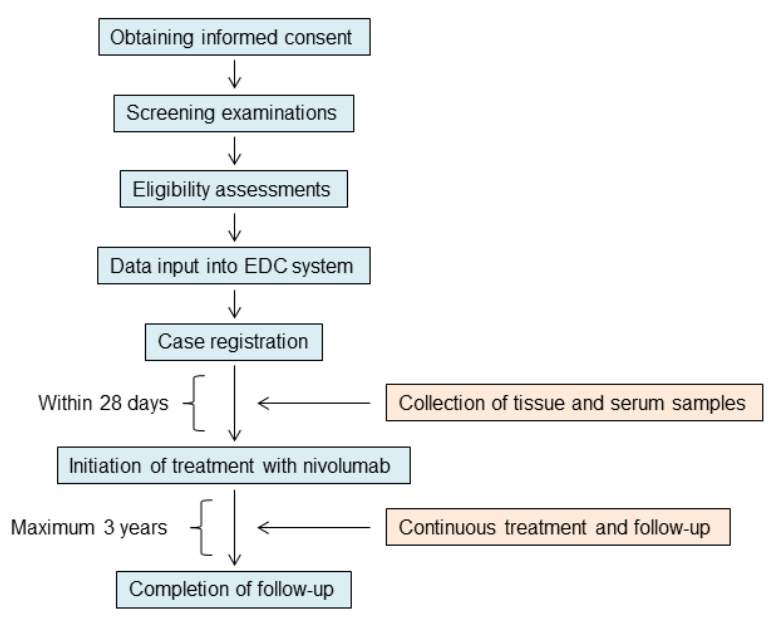

Figure 1: Schedule for the URivo study.

\section{DISCUSSION}

The recent introduction of immune checkpoint inhibitors into clinical practice has resulted in marked changes in the therapeutic strategy for patients with aRCC. ${ }^{5}$ In particular, nivolumab is currently regarded as a standard agent for aRCC patients previously received antiangiogenic therapies based on the results of the CheckMate 025 trial, demonstrating a significantly superior OS in previously treated aRCC patients receiving nivolumab to those receiving everolimus. ${ }^{7}$ Although the marked benefit of nivolumab has been widely accepted, there are several problems associated with the use of this agent for aRCC patients, such as the variable response rates and development of immunerelated AEs. ${ }^{6}$ To date, however, there have been no biomarkers to precisely predict the clinical course of aRCC patients treated with nivolumab; therefore, the URivo study was designed to examine the utilities of several candidate biomarkers in aRCC patients who will receive nivolumab following the failure of either 1 or 2 TKIs. 5

Considering the mechanisms of action of immune checkpoint inhibitors blocking the binding of PD-1 to its ligands, PD-L1 could theoretically be expected to be a useful biomarker for aRCC patients receiving these agents. ${ }^{11}$ However, similar to other types of malignant tumor, the role of PD-L1 expression by tumor cells and/or immune cells to predict treatment outcomes of immune checkpoint inhibitors, including nivolumab, in patients with aRCC remains unclear. ${ }^{10}$ For example, a prognostic benefit was shown for aRCC patients treated with nivolumab irrespective of the expression of PD-L1 in tumor tissues in the CheckMate 025 trial. $^{7}$ The present inconsistencies regarding the significance of PD-L1 expression as a biomarker for patients treated with immune checkpoint inhibitors could be explained by several factors, such as the use of different antibodies for immunohistochemical staining, lack of a definitive cut- 
off reference for PD-L1 positivity and various patterns of the types of cells on which PD-L1 expression is evaluated. ${ }^{11}$ Collectively, these findings strongly suggest that it is important to comprehensively assess the predictive value of PD-L1 in aRCC patients receiving nivolumab; therefore, the copy number of the $P D-L 1$ gene, serum concentration of PD-L1 and PD-L1 protein expression will be assessed by FISH, ELISA and immunohistochemical staining, respectively, in this study. In particular, it will be of interest to investigate the impact of $P D-L 1$ copy number gains on the clinical course of aRCC patients treated with nivolumab, since a recent study by Inoue et al reported that an increase in the $P D-L 1$ gene copy number examined by FISH could be a more feasible alternative biomarker than PD-L1 protein expression for predicting the response to anti-PD-1/PDL1 therapy. ${ }^{13}$

Another point of interest is the exploration of candidate biomarkers showing no association with the action mechanism of immune checkpoint inhibitors. To date, several studies indicated the more aggressive nature of tumors with PD-L1 expression than that of those without PD-L1 expression in RCC. ${ }^{11}$ For example, aRCC patients with tumors showing $\geq 1 \%$ PD-L1 expression were shown to have significantly shorter OS than those with tumors $<1 \%$ PD-L1 expression in the CheckMate 025 trial. $^{7} \mathrm{We}$ also previously analyzed the expression pattern of immune checkpoint-associated molecules in tumor tissues in patients with aRCC treated with TKIs, and showed that patients with positive PDL-L1 expression had significantly unfavorable PFS and OS compared with those without positive PD-L1 expression. ${ }^{14}$ Taken together, traditional molecular biomarkers involved in the malignant progression of RCC may have significant impacts on prediction of the clinical course of aRCC patients receiving nivolumab; thus, the expression profile of major proteins mediating the apoptosis, epithelialmesenchymal transition (EMT) and signal transduction in addition to immunological reaction in tumor tissues will be assessed by immunohistochemical staining in this study.

Here, we would like to describe several limitations of the protocol of the URivo study. Initially, it would be ideal to perform genetic examinations of some other candidate biomarkers involved in the response to immune checkpoint inhibitors. For example, it may be useful to assess the status of neoantigens, mutations encoding immunologically active proteins that can be functional targets of immune checkpoint inhibitors and lead to a response to these agents. ${ }^{15}$ Secondly, more detailed evaluation of the immunomodulatory activity of nivolumab, such as tumor-associated lymphocytes and serum chemokines, may provide insight into the discovery of biomarkers for this agent. ${ }^{16}$ Finally, it will be necessary to carefully consider the current as well as future marked changes in first-line therapy for aRCC by the introduction of immune checkpoint inhibitor-based combined regimens, when reviewing the protocol of this study. ${ }^{17}$

As discussed above, despite several potential biomarkers being examined to predict the response to nivolumab, none have been introduced into clinical practice for aRCC patients through prospective validation. We believe that the outcomes of this proposed study will help identify biomarkers to predict the likelihood of aRCC patients benefiting from treatment with nivolumab. Particularly, comprehensive assessments of the significance of the PD-L1 expression status by multiple assays may yield definitive information with respect to whether the investigation of this molecule will provide useful information for the selection of aRCC patients to receive novolumab.

\section{ACKNOWLEDGEMENTS}

We thank all participating institutions, including the nursing and physician staff.

Funding: The biomarker research costs for the URivo study is partially supported by ONO PHARMACEUTICAL CO., LTD (Osaka, Japan) and Bristol-Myers Squibb K.K. (Tokyo, Japan)

Conflict of interest: None declared

Ethical approval: The study was approved by the Institutional Ethics Committee

\section{REFERENCES}

1. Randall JM, Millard F, Kurzrock R. Molecular aberrations, targeted therapy, and renal cell carcinoma: current state-of-the-art. Cancer Metastasis Rev. 2014;33(4):1109-24.

2. Minguet J, Smith KH, Bramlage CP, Bramlage P. Targeted therapies for treatment of renal cell carcinoma: recent advances and future perspectives. Cancer Chemother Pharmacol. 2015;76(2):219-33.

3. Bedke J, Gauler T, Grünwald V, Hegele A, Herrmann E, Hinz S, et al. Systemic therapy in metastatic renal cell carcinoma. World J Urol. 2017;35(2):179-88.

4. van der Mijn JC, Mier JW, Broxterman HJ, Verheul HM. Predictive biomarkers in renal cell cancer: insights in drug resistance mechanisms. Drug Resist Updat. 2014;17(4-6):77-88.

5. Atkins MB, Clark JI, Quinn DI. Immune checkpoint inhibitors in advanced renal cell carcinoma: experience to date and future directions. Ann Oncol. 2017;28(7):1484-94.

6. Mennitto A, Grassi P, Ratta R, Verzoni E, Prisciandaro M, Procopio G. Nivolumab in the treatment of advanced renal cell carcinoma: clinical trial evidence and experience. Ther Adv Urol. 2016;8(5):319-26.

7. Motzer RJ, Escudier B, McDermott DF, George S, Hammers HJ, Srinivas S, et al. Nivolumab versus 
everolimus in advanced renal-cell carcinoma. N Engl J Med. 2015;373(19):1803-13.

8. Tannir NM, Pal SK, Atkins MB. Second-line treatment landscape for renal cell carcinoma: a comprehensive review. Oncologist. 2018;23(5):54055.

9. Ribas A, Wolchok JD. Cancer immunotherapy using checkpoint blockade. Science. 2018;359(6382):1350-5.

10. Gibney GT, Weiner LM, Atkins MB. Predictive biomarkers for checkpoint inhibitor-based immunotherapy. Lancet Oncol. 2016;17(12):e54251.

11. Festino L, Botti G, Lorigan P, Masucci GV, Hipp JD, Horak CE, et al. Cancer Treatment with AntiPD-1/PD-L1 Agents: Is PD-L1 Expression a Biomarker for Patient Selection? Drugs. 2016;76(9):925-45.

12. Motzer RJ, Bacik J, Schwartz LH, Reuter V, Russo $\mathrm{P}$, Marion S, et al. Prognostic factors for survival in previously treated patients with metastatic renal cell carcinoma. J Clin Oncol. 2004;22(3):454-63.

13. Inoue Y, Yoshimura K, Mori K, Kurabe N, Kahyo $\mathrm{T}$, Mori $\mathrm{H}$, et al. Clinical significance of PD-L1 and PD-L2 copy number gains in non-small-cell lung cancer. Oncotarget. 2016;7(22):32113-28.
14. Hara T, Miyake H, Fujisawa M. Expression pattern of immune checkpoint-associated molecules in radical nephrectomy specimens as a prognosticator in patients with metastatic renal cell carcinoma treated with tyrosine kinase inhibitors. Urol Oncol. 2017;35(6):363-9.

15. Riaz N, Morris L, Havel JJ, Makarov V, Desrichard A, Chan TA. The role of neoantigens in response to immune checkpoint blockade. Int Immunol. 2016;28(8):411-9.

16. Choueiri TK, Fishman MN, Escudier B, McDermott DF, Drake CG, Kluger H, et al. Immunomodulatory activity of nivolumab in metastatic renal cell Carcinoma. Clin Cancer Res. 2016;22(22):5461-71.

17. Choueiri TK, Motzer RJ. Systemic therapy for metastatic renal-cell carcinoma. $\mathrm{N}$ Engl J Med. 2017;376(4):354-66.

Cite this article as: Miyake $\mathrm{H}$, Harada $\mathrm{K}$, Matsushita Y, Hinata N, Sugimura H, Fujisawa M. Protocol for a prospective multicenter intervention study (URivo study) assessing biomarkers in patients with previously treated advanced clear cell renal cell carcinoma receiving nivolumab. Int J Clin Trials 2019;6(2):63-8. 